\title{
LA RADICALIZACIÓN DEL ÉLENCHOS: EL RECURSO A LA VERDAD COMO CONDICIÓN DIALÉCTICA MÍNIMA EN EL TEETETO DE PLATÓN
}

Pilar Spangenberg

UBA - CONICET

pspangenberg@hotmail.com

En la primera parte del Teeteto Sócrates refuta tres tesis que considera estrechamente vinculadas entre sí: la tesis de Teeteto que identifica conocimiento y percepción, la tesis relativista y la tesis movilista. El propósito de este trabajo es mostrar que en tres de las refutaciones ofrecidas contra estas posiciones se exhibe una misma estrategia tendiente a remitir a un prerrequisito dialéctico vinculado a la pretensión de verdad. Esta constituiría una estrategia radical, en el sentido de que pone en cuestión la misma posibilidad de emitir la propia tesis por parte del adversario.

Platón / élenchos / relativismo / verdad / dialéctica

THE RADICALIZATION OF THE ÉLENCHOS: THE RECOURSE TO TRUTH AS MINIMAL DIALECTICAL CONDITION IN THE THEAETETUS OF PLATO.

In the first part of Theaetetus Socrates refutes three theses that he considers intimately linked: the one that identifies knowledge and perception, the relativistic thesis and another about mobilism. The purpose of this work is to show that in three of the refutations offered against these positions the same strategy is displayed in order to refer to a dialectic prerequisite linked to a truth claim. This would constitute a radical strategy due to the fact that it questions the same possibility of expressing its own thesis by the adversary.

Plato / elenchos / relativism / truth / dialectic 
$\mathrm{E}$

n la primera parte del Teeteto, en su afán de definir el conocimiento (epistéme), Sócrates postula una fuerte interconexión entre tres tesis: la tesis de la identidad entre conocimiento y percepción, la de la homo-mensura atribuida a Protágoras y la del heraclitismo, que habría identificado ser y movimiento. Si bien Sócrates establece que tales tesis se implican mutuamente, cada una de ellas es refutada por separado. En primer lugar, Sócrates refuta la tesis de Protágoras ofreciendo, entre otros argumentos, el de la célebre auto-refutación; en segundo lugar, refuta el movilismo extremo sirviéndose de un argumento que acude directamente a la imposibilidad de presentar la propia tesis por parte del adversario; $y$, por último, refuta la tesis de la identificación entre conocimiento y percepción, acudiendo a un argumento que pretende exhibir que todo conocimiento supone la aprehensión del ser y de la verdad y que tal aprehensión no puede darse en el nivel de la percepción, sino que constituye una actividad que el alma realiza por sí misma al margen de los sentidos.

En este trabajo interesa mostrar que, a pesar de constituir refutaciones independientes, la estrategia asumida en todas ellas supone un patrón común explícito en las dos primeras, implícito en la última: el recurso a la pretensión de verdad en tanto condición de enunciación de todo discurso lanzado a la arena dialéctica ${ }^{1}$. En efecto, intentaré mostrar que en cada uno

Asumo en este trabajo una intrínseca relación entre dialéctica y refutación (élenchos) en lo que concierne, al menos, al Teeteto. Por dialéctica entiendo aquí el método filosófico del que se vale Platón para describir el peculiar modo de acercamiento teórico del filósofo a su objeto. Como destaca RoBinson (1941: 73-74), a pesar de las diversas formas que éste fue asumiendo a lo largo de su obra, siempre constituyó para Platón el método ideal y, más allá de sus diversas versiones, fue adjudicado por él en todos los casos al verdadero filósofo. Desde el punto de vista formal e intentando una aproximación general, supone un diálogo que va avanzando a partir de preguntas y respuestas entre dos personajes que se abocan al examen de una noción determinada (NiкULiN 2010: 4-6). En el período temprano de la obra platónica la dialéctica puede ser identificada con la refutación (élenchos). Ella refiere al examen o interrogatorio realizado por Sócrates a un interlocutor en relación con una afirmación inicial por él formulada con el fin de determinar su sentido y valor de verdad. Para hacerlo, Sócrates va planteando sucesivas preguntas que requieren nuevas respuestas que conducen finalmente a exhibir la falsedad de la afirmación inicial a través de la constatación de la contradicción en que el interlocutor ha incurrido en sus sucesivas respuestas (RoBINSON 1941: 7). Aun cuando, es en el período temprano de la obra platónica que la refutación (élenchos) asume un lugar preponderante al punto de identificarse con la dialéctica, el Teeteto -diálogo del período tardío en el cual la dialéctica se caracterizará fundamentalmente por una búsqueda de la definición en función de la doble operación de la reunión y la división- se asimila en algunos aspectos a los diálogos de juventud. Estos aspectos, desde mi punto de vista, son dos: en primer lugar, que el diálogo avanza fundamentalmente a través del élenchos (si bien, a diferencia de los diálogos tempranos, no se trata de una única refutación la que está en juego, sino de un entramado complejo que se estructura a partir de tres refutaciones principales a las tres definiciones sucesivas que

ARgos 39 (2016) ISSN 0325-4194, pp. 9-32 
de ellos opera como condición algo que podríamos denominar "prerrequisito de pretensión de verdad", a partir del cual el acto de la enunciación en un contexto dialéctico invalida el contenido de cada una de las tres tesis en cuestión. No aspiro, entonces, a ofrecer una lectura pormenorizada de cada uno de los argumentos mencionados, pero sí a exhibir ciertos hilos invisibles que desde la mirada platónica ligarían las tres tesis y que podrían iluminar la mecánica general de los argumentos en cuestión.

\section{La crítica a Protágoras: la peritropé (169d3-171e9)}

En el marco del análisis de la primera respuesta ofrecida por Teeteto a la pregunta “¿qué es el conocimiento (epistéme)?" que vertebra el diálogo, Sócrates traza una suerte de entramado de teorías que, según da a entender, se implican la una a la otra ${ }^{2}$. Es un entramado complejo cuyas tres aristas principales son, por un lado, la tesis de la identificación entre conocimiento y percepción defendida inicialmente por Teeteto y que oficia de punto de partida de todo el análisis de la primera parte del diálogo; en segundo lugar, la tesis relativista de Protágoras representada en la sentencia de la homo-mensura y, por último, una tesis de pretendido cuño heraclíteo según la cual el ser se identifica con el movimiento. Luego de tejer el entramado teórico a partir de un análisis pormenorizado de la respuesta de Teeteto, Sócrates abre en 160 e el momento crítico del análisis y embiste con diversas críticas frente a cada una de las tres tesis mencionadas ${ }^{3}$. Comienza por la posición relativista defendida por Protágoras. Después de ofrecer una crítica dirigida al igualitarismo de las opiniones propiciado por dicha posición (161b-163a) y una serie de objeciones a la identificación entre conocimiento y percepción (163a-165d), cree saldar la discusión con Protágoras en lo que concierne al llamado "protagorismo amplio", es decir, la tesis según la cual toda dóxa sea cual fuere su objeto es verdadera, acudiendo al célebre argumento de la auto-refutación (170a5-171c6), apodado por Sexto argumento de la peritropét. . El argumento, presentado en sus líneas más gruesas, sostiene que si toda opinión es igualmente verdadera,

se ofrecen del conocimiento (epistéme $)$ ); y, en segundo lugar y estrechamente vinculado al punto recién señalado, que es un diálogo aporético: si bien Sócrates se asienta en muchas posiciones a la hora de refutar las tesis analizadas, no responde finalmente a la pregunta que guía el diálogo -¿qué es el conocimiento?-.

2 Con respecto a este entramado de teorías y de la relación de mutua implicación que les atribuye Sócrates cf. LeE (2005: 86-87) y SPANGENBERg (2009).

3 Lo cual nos ha conducido a pensar que la implicación pretendida entre ellas no termina de resultarle del todo convincente ni siquiera al propio Sócrates.

4 Esta calificación se la otorga Sexto Empírico en Adv. Math. VII 389. Respecto de las diferencias con el presente argumento cf. BuRNYEAT (1976) y en cuanto a la distinción 
Protágoras deberá reconocer que también lo es la de la mayoría, que considera que su propia doctrina es falsa. Esta es la conclusión del argumento:

Entonces, esta [la Verdad escrita por Protágoras] tiene su característica más sutil: aquel, por su parte, acordando (homologônn) que todos opinan lo que es, deberá admitir (sunchoreî) que es verdadera la creencia (oíesin) de los que mantienen creencias contrarias a la suya (tệs autoû oiéseôs), como ocurre en el caso de los que consideran que él está equivocado. ¿Entonces él debería admitir (sunchorôi) que su propia opinión es falsa, si acuerda (homologeî) que es verdadera la de aquellos que mantienen que él está equivocado? (...) Pero los otros no admiten (ou sunchoroûsin) que ellos mismos estén equivocados. (...) Mientras que él acuerda (homologeî) que la opinión de ellos es verdadera, según lo que escribió. (...) Entonces todos, empezando por Protágoras, cuestionarán (amphisbetésetai) esta opinión, y él acordará (homologésetai) con la posición de sus oponentes: pues admite (sunchorêii) que la opinión de quienes dicen lo contrario de él es verdadera. (Teeteto 171a6-b12) ${ }^{5}$

El esqueleto del argumento puede ser reconstruido en estos términos: Si (A) toda opinión es verdadera,

$\mathrm{y} \quad$ (B) (A) es considerado falso,

entonces $(C)$ es verdadero que $(A)$ es falso

$\mathrm{y}$, consecuentemente, (D) (A) es falso ${ }^{6}$.

En este polémico argumento opera a todas luces una noción de verdad que, según han sostenido muchos comentadores, está implícitamente negada en la teoría de Protágoras, en la medida que supone la ausencia de relativizadores. En efecto, parecería que si respetamos el relativismo defendido por el sofista, el argumento de la peritropé propuesto por Platón no alcanza a afectar la tesis de la homo-mensura, pues su autor no admitiría (A), es decir una concepción de la verdad simpliciter en la que se excluyen los relativizadores ${ }^{7}$. Así, Protágoras puede aceptar la verdad de la posición del adversario que niega la tesis de la verdad de toda opinión, sin

entre un protagorismo amplio y uno estrecho cf. CROMBIE (1963: 13 y ss.) y FINE (1994: 213-214).

5 Las traducciones son propias. He utilizado la edición de Duke, E. A., Hicken, W. F. And Nicoll, W. S. M. et al. (1995).

6 Respecto de esta reconstrucción y los problemas que suscita el paso (A) cf. BURNYEAT (1976: 40-41).

7 Así se sigue de 152b-c, 158a, 166c-167c, 170c y 171e y 172a, pasajes en que invariablemente Protágoras presenta su posición acerca de la verdad utilizando los relativizadores. Como señala VLASTOS (1956: XIV n. 29), en algunos casos la meticulosidad 
por eso comprometerse con su posición ni admitir la falsedad de su propia tesis $^{8}$. La adición de los relativizadores bloquearía la crítica, pues el sofista no tiene por qué adoptar el vocabulario no relativizado de sus oponentes.

Frente a esta crítica se han dado diversas respuestas. Es claro que Platón no desconoce la relevancia de los relativizadores en el pensamiento de Protágoras, ¿por qué, entonces, los pasó por alto en este argumento? Se podría pensar que Platón no se está sirviendo del "contenido" de la tesis protagórica, sino de lo que se sigue del mero hecho de que haya postulado su tesis ${ }^{9}$. En este sentido, resulta particularmente importante reparar en la profusión de términos propios del campo dialéctico que aparece en el pasaje citado. En efecto, la presencia de los términos "homologeîn y sunchoreîn" está acentuando la dimensión dialéctica en que se pretende situar el argumento. "Homologeîn" refiere al acuerdo, al convenio, a una condición básica dialéctica según la cual cualquier progresión en la discusión o el diálogo supone el acuerdo de sendas partes en los puntos hasta allí establecidos ${ }^{10}$. "Sunchoreîn", en cambio, refiere a un momento de repliegue en el campo de batalla, a la necesidad de ceder terreno. En ese sentido pone de manifiesto otra cara de la dialéctica que se verifica también desde los primeros diálogos: los interlocutores muchas veces se ven constreñidos, en función de sus propias afirmaciones, a admitir, aún contra su deseo, lo que se sigue de tales afirmaciones ${ }^{11}$. Así, Sócrates estaría

de Sócrates en la adición del "para ..." a la hora de dar cuenta de la posición de Protágoras resulta hasta tediosa.

8 Sobre este aspecto de la peritropé cf. BuRnYEAT (1976: 41), WATERLOW (1977: 29-30), Bostock (1988: 89-90), LEE (2005: 48-57), Fine (1994: 213-214), CASTAGNOLI (2010: 46).

9 Como señala CASTAGnOLI (2010: 32), en lo que concierne a los argumentos auto-refutativos es incorrecto considerar que pueden ser desprovistos de su ropaje dialéctico originario y traducidos a la forma monológica sin malinterpretarlos. Pues, de acuerdo con el punto de vista aquí adoptado, el elemento que se contradice con la propia tesis es justamente el hecho de formularla en un contexto dialéctico, lo cual conlleva necesariamente la pretensión de defender la verdad de la propia tesis y, a su vez, demostrar la falsedad de la tesis contradictoria. Eso, según Castagnoli (2010: 66), es lo que Protágoras no puede hacer a la luz del contenido de su propia tesis, pero, a su vez, y de eso se vale Sócrates, no puede dejar de hacerlo en la medida que se presta al juego dialéctico.

10 Con respecto a este requisito dialéctico cf. Robinson (1941: 15). Este se establece explícitamente, por ejemplo, en Gorgias 471d.

11 Al respecto cf. el excelente tratamiento que acerca de esta terminología dialéctica desarrolla MArcos (2006: 214-218). Allí defiende la tesis de que mediante el empleo de los verbos homologeîn y sunchoreîn Platón estaría intentando marcar un contraste entre aquellas tesis que Protágoras defiende explícitamente y aquello que sobre la base de lo afirmado, se ve forzado a conceder a sus oponentes en virtud de sus propias admisiones (no, como considera la mayoría de los estudiosos, de las de otro). Así, subraya el hecho de que la distinción entre homologeîn y sunchoreîn se correspondería aproximadamente con aquella 
indicando a través del encadenamiento de estos términos que, en función de sus propias afirmaciones, Protágoras no puede sino acordar con su adversario: la identidad del sofista se desdibuja en la imposibilidad de diferenciarse de un otro en el campo dialéctico ${ }^{12}$. En virtud de su propia tesis, Protágoras no puede introducirse en esta especial dinámica de identidad y diferencia que supone toda dialéctica. Sin embargo, Protágoras lanzó su tesis con alguna pretensión de validez y su postulación, sugeriría Platón, lo compromete al margen del contenido de sus tesis, con una pretensión de verdad simpliciter. En otros términos, si el sofista reintrodujera los relativizadores quedaría inmunizado frente a la crítica de la necesaria identificación con el adversario, pero tal inmunidad debería pagar un alto precio, según parece pensar Platón: el Abderita no estaría diciendo nada realmente serio, que pueda discutirse o negarse de un modo significativo, y quedaría, por consiguiente, cercado por sus propios relativizadores ${ }^{13}$. No tendría así ningún sentido discutir su tesis. Como contraparte, entonces, habría que afirmar que el factum de la exposición de la tesis en la arena dialéctica lo compromete ya con una pretensión de verdad, cuya negación dejaría desprovisto de todo sentido el solo hecho de someter el propio discurso a discusión ${ }^{14}$. De modo que, lejos de no haber percibido esta condición, Platón estaría implicando en su crítica la necesidad de someterse a reglas dialécticas mínimas en la medida en que se quiera defender una cierta posición teórica, como la de la homo-mensura establecida por Protágoras ${ }^{15}$. Esta

que se da entre lo expreso y lo tácito. Es por eso que una misma proposición es introducida a veces por sunchoreîn y a continuación por homologeîn, pues lo que era conclusión de un tren de razonamiento oficia de premisa del siguiente. Por otra parte, interesa señalar que la relación de complementariedad que en este pasaje se verifica entre ambos términos nos da la pauta de que la dialéctica no puede prescindir en ningún caso de cierta dimensión confrontativa, la cual, frente al empleo que de ella hace la erística, constituiría el motor necesario de cualquier examen.

12 Sócrates, de hecho, refiere a la imposibilidad de conciliar la tesis de Protágoras con cualquier pretensión dialéctica al afirmar: "Guardo silencio sobre mí mismo y el arte de partero que practico, y cuán ridículo resulta este; y creo que lo mismo sucede respecto de la práctica dialéctica (dialégesthai) en su totalidad. Pues si La Verdad de Protágoras es verdadera ¿no sería un gran y enorme sinsentido examinar e intentar refutar (elénchein) los pareceres y opiniones de unos y otros al ser acertadas las de cada uno?" (Teet. 161e4-162a2)

13 Con respecto a esta posición cf. LEE (1973: 245-246) y especialmente MARCos (1996) y (2006).

14 En ese sentido, como señala MARcos (1996: 435-436), se explican las recurrentes referencias a la voluntad protagórica de someter su tesis a discusión. Al respecto cf. Teet. 165e-166a, 167d-168b, 168c-169d.

15 En palabras de Burnyeat (1976: 58-59): "si Protágoras postula su relativismo y admite que esta doctrina (...) es verdadera para él, debe reconocer también que es una verdad absoluta que la doctrina de la Medida es verdadera para Protágoras." Y poco más adelante 
presunción, a su vez, sería admitida por la mayoría al establecer como falsa la tesis de la verdad de toda opinión.

Así, según hemos establecido, es posible considerar que la posición de Protágoras no puede ser refutada en sentido estricto, pues ante cualquier crítica que se formule contra ella es posible reintroducir los relativizadores: se la acepta o se la rechaza, pues se yergue en tanto principio. Su peculiaridad residiría en que desde las categorías internas de su sistema no es posible destruirla. Platón parece haber sido consciente de esta peculiar condición de la tesis relativista y por esa razón, a la hora de refutarla habría remitido a condiciones anteriores a las establecidas por la tesis. En consecuencia, podría pensarse que la efectividad del tipo de estrategia del que se sirve contra ella residiría en el hecho de mostrar, desde condiciones previas al sistema de pensamiento del adversario, que su propia tesis conlleva la imposibilidad de cualquier diálogo posible ${ }^{16}$. En los términos de Waterlow, el objetivo último de Platón es mostrar que un oponente que se enfrentara a la posición de Protágoras "confrontaría, por decirlo de alguna manera, con una nada dialéctica, que no podría ofrecer ningún tipo de resistencia" ${ }^{17}$. Esta imposibilidad se pone en evidencia cuando Sócrates, burlonamente señala que Protágoras podría regresar:

afirma: "Ninguna maniobra con los relativizadores excluirá a Protágoras del compromiso con la verdad absoluta que está implicado en el mismo acto de la aserción".

16 Según considera WATERLow (1977: 26), sería absurdo que Platón hubiera buscado refutar a Protágoras exhibiendo la inconsistencia en la que incurriría, porque de hecho en dos pasajes parece presentarlo como afirmando la posibilidad (y la necesidad en sentido estricto) de la contradicción. Al respecto cf. 166b4-5 y 167a7-8. ¿Qué sentido tendría, entonces, pretender refutarlo acudiendo a puntos de partida ajenos a los admitidos por Protágoras? Frente a la posición de Waterlow, considero que la prueba no apunta a refutar al sofista demostrando que él afirma y niega lo mismo, sino más bien que tiene que admitir la falsedad de su tesis por haber establecido que toda opinión es igualmente verdadera. Esto no implica rechazar que su posición conduzca a contradicciones, pero ¿para qué probar que es falsa por contradictoria si ya probó (y Protágoras tuvo que admitir) que es falsa? WATERLOW señala que hay una segunda razón por la cual Platón debería haber considerado ineficiente cualquier refutación de la posición de Protágoras acudiendo a la inconsistencia: para Protágoras, la contradicción ocurre sólo cuando es el mismo sujeto quien sostiene creencias contradictorias. Y siendo el Protágoras del Teeteto un heraclíteo, afirmaría que un sujeto nunca es el mismo de un instante a otro. Cualquier sujeto denota, entonces, una ficción. Esta segunda razón no me resulta del todo convincente porque entiendo que aun cuando a lo largo de la primera parte Platón traza esta equivalencia entre las dos tesis, a la hora de la refutación ofrece un tratamiento independiente de cada una de ellas.

17 WATERLow (1977: 36). Interesa señalar que si bien comparto esta conclusión, no acuerdo respecto del modo en que la autora arriba a ella, pues intenta demostrar que el relativismo enunciado por Protágoras no es un relativismo sobre la verdad sino un relativismo ontológico, en el sentido que considera que lo que es relativo a cada uno es el mundo y no la verdad. En este sentido, lo que están en juego son verdades simpliciter y no tiene 
Y si sacara su cabeza de la tierra hasta el cuello, él seguramente me refutaría (elénchas) a mí por decir sinsentidos y a ti por acordar conmigo, y entonces se iría corriendo y se ocultaría. Pero yo creo que nosotros tenemos que tratar con nosotros mismos tal como somos y decir siempre lo que nos parece. (171c10-d5)

En el pasaje Sócrates no solamente alude a la imposiblidad por parte de Protágoras de llevar a cabo refutación o acusación alguna -esta sería la razón por la cual el sofista se daría a la fuga avergonzado y se volvería a sumergir en la tierra- sino que además, frente a la figura inconsecuente de Protágoras, defiende la necesidad de mostrarse tal cual es y de decir siempre lo que cree. En este sentido, y dando una vuelta de tuerca a la estrategia que guía el argumento, podríamos afirmar que, siendo imposible una refutación desde el interior de la tesis protagórica, la prueba, que opera sobre el trasfondo del recurso a los prerrequisitos dialécticos de pretensión de verdad, tendría una dimensión ad hominem, pues según se insinúa en el pasaje recién citado, quien pretendiera defender una posición como la protagórica por el hecho mismo de lanzar su tesis en la arena dialéctica exhibiría que ni él mismo cree lo dicho, en la medida que el hecho mismo de afirmar una tesis -y, más aún, defenderla como pretende hacer Protágoras saliendo de la tierra-se asienta en la confianza en la posiblidad de la falsedad ${ }^{18}$.

\section{La crítica al heraclitismo (179c1-183c7)}

Sócrates no se da por satisfecho con la refutación recién examinada y considera que es necesario ofrecer una nueva, pues "acerca de las impresiones presentes de cada uno, a partir de las cuales se generan las opiniones y percepciones correspondientes, es más difícil probar que no son verdaderas" (179c2-5). Sócrates arremeterá, entonces, contra la tesis heraclítea que identificaba ser y movimiento, por constituir la base metafísica sobre la cual Protágoras en su doctrina secreta había establecido la verdad de toda percepción ${ }^{19}$. Recién al finalizar el argumento considerará

sentido recurrir a los relativizadores. Protágoras podría admitir así que la posición de sus oponentes es verdadera simpliciter, pero es falsa para él por no compartir la misma realidad respecto de la cual tal afirmación es verdadera.

18 Esta sería la razón por la cual, como señala CAstagnol (2010: 66-67), Protágoras no puede reaccionar ni siquiera frente a las reformulaciones socráticas de su tesis que dejan de lado los relativizadores como sucede en 170c2-4.

19 Esta compleja teoría se ofrece en varias versiones a lo largo de la primera parte del diálogo. Cf. Teet. 153d-154b; 155d-157c; 158e-160e; 181b-182b. 
acabada la discusión con Protágoras (183b7-9)20. La refutación socrática establece la imposibilidad de enunciar la propia tesis por parte de los heraclíteos, quienes no dispondrían de expresiones adecuadas para hacerlo, pues a partir de la concepción que intentan defender, según la cual todas las cosas están en continuo movimiento en cualquier sentido posible, en el momento de decir cualquier cosa, ella se escabulliría por estar inmersa en el flujo (182d1-4). Ahora bien, interesa aquí mostrar que esta imposibilidad de enunciar la propia tesis por parte del adversario no se debe a un colapso del lenguaje como estima la mayoría de los intérpretes, sino más bien a la imposibilidad de un lenguaje que pretenda determinar la naturaleza de las cosas. En otras palabras, aquello que se pone en tela de juicio a partir de la tesis movilista no es la posibilidad del lenguaje como tal, sino la del lenguaje requerido en el juego dialéctico. Así, lo que se pone en duda en esta prueba no es tanto la misma posibilidad de hablar, sino la de ofrecer un discurso verdadero ${ }^{21}$. Probablemente haya sido el aire de familia que lo liga con la primera refutación que ofrece Aristóteles contra el negador del principio de no contradicción en Metafisica Gamma 4, 1006a281007a21, la que ha conducido a pensar que aquello que intenta exhibir Platón en este argumento es un colapso del lenguaje. En Aristóteles, en efecto, la posición heraclítea sí conlleva la imposibilidad de hablar, pues la que se ve comprometida es la posibilidad de la significación. Junto con la imposibilidad de la determinación que conlleva la negación del principio de no contradicción cae la posibilidad de la significación. Pero aquí, según intentaré mostrar, no es la posibilidad del lenguaje la que está en juego, sino la de la verdad supuesta en el acto de la enunciación de cualquier tesis en un escenario dialéctico.

De hecho, en 179 e antes de presentar el argumento ya en cierto modo se avanza el blanco de la crítica al afirmar la imposibilidad de mantener un intercambio dialéctico (dialechthênai, 179e6-7) con el heraclíteo. En

20 "Pues bien Teodoro, ya nos hemos librado de tu amigo, y sin embargo no le hemos concedido que todo hombre sea medida de todas las cosas, salvo que sea alguien prudente".

21 CoRnFord (1935: 96-101) interpretó este argumento a partir de su consideración general de que la teoría del flujo establecida aquí es suscripta por Platón en relación con lo sensible. En este sentido, considera que es una prueba de que no todo puede moverse: en tal caso los términos no podrían tener un significado fijo. Si las palabras tienen un significado fijo es porque dependen de las formas, y si estas también estuvieran sometidas al movimiento, todo lenguaje sería imposible y nada podría afirmarse. Cornford considera que cuando Platón se refiere a la blancura, por ejemplo, se refiere a una idea. En consecuencia, las cosas sensibles están en movimiento, pero las formas no. Contra esta posición cf. Bostock (1988: 103), quien ha sido muy convincente al defender la imposibilidad de considerar que esta teoría extrema del flujo haya podido ser defendida por Platón aun respecto de lo sensible. 
efecto, allí Teodoro, con evidente sorna, refiriéndose a los que defienden una teoría extrema del flujo, afirma que, puesto que la gente de Éfeso se encuentra continuamente en movimiento, es más difícil dialogar con ellos que con maniáticos. Y a continuación afirma:

Y la posibilidad de detenerse en lo que se refiere a un argumento o una pregunta (epi lógôi kai erótémati), esperando tranquilamente para responder o hablar en turnos (en mérei apokrinasthai kai erésthai), es absolutamente nula. (...) Si le preguntas (éreii) a uno, como si fuera una flecha que hubiera sacado de su carcaj, te dispara un aforismo enigmático $\mathrm{y}$, si buscas que te dé una explicación (lógon) de qué ha dicho (ti eiréken), te alcanzará con una nueva expresión en que habrá cambiado totalmente las palabras (metonomasménoi i). (...) Se cuidan bien de no permitir que haya nada estable ni en el discurso (en lógoi $i$ ni en sus propias almas porque, según me parece, piensan que eso sería algo inmóvil. Y, sin embargo (dé), contra esto polemizan mucho (pánu polemoûsin), pues su intención es suprimir [lo inmóvil] tan absolutamente como sea posible (179e9-180b2).

Al margen de la evidente ridiculización de la que es objeto la figura del heraclíteo, del discurso de Teodoro surge con claridad la imposibilidad de mantener un diálogo con un personaje tal. La referencia a la constelación semántica dialéctica es evidente. Se le niega al movilista la posibilidad de mantener un diálogo, pues no puede preguntar ni responder por turnos. Tampoco es posible solicitarle ningún tipo de explicación acerca de lo que él mismo dice, porque enseguida cambia sus palabras. Nada estable puede encontrarse en su discurso. Y, sin embargo, afirma Teodoro, el movilista pretende polemizar contra cualquier posición que afirme la posibilidad de algún tipo de estabilidad. La oposición que aquí se verifica en 180b1 a través de la conjunción adversativa "dé" guarda especial relevancia, desde mi punto de vista, puesto que es en el hiato que media entre el uso indefinido y ambiguo del lenguaje y la pretensión de defender una cierta tesis en el contexto dialéctico donde Platón encontrará el espacio para convertir en una nada dialéctica también al movilista. Vamos, entonces, al análisis del argumento en sus trazos más generales para exhibir el modo en que se despliega la mencionada estrategia contra el movilista.

En 181c-d Sócrates distingue dos tipos de cambio: traslación (phorá) que es el tipo de movimiento del que se sirvió hasta ahora el heraclíteo para dar cuenta de la percepción, y alteración (alloíosis), que se trataría del cambio cualitativo. Los heraclíteos afirman, según establece Sócrates y admite Teeteto en 181e10-182a4, que todo está sometido al cambio en am- 
bos sentidos, pues no puede haber inmovilidad alguna; el movimiento debe ser absoluto (teléós kinésetai,182c8). En este contexto Sócrates afirma:

lo blanco (tò leukón) que fluye no permanece blanco en su fluir, sino que cambia de modo que el flujo también afecta a esto mismo, a la blancura, y hay cambio hacia otro color, para que no se le pueda condenar por permanecer de este modo. En consecuencia, ¿podríamos acaso asignarle (proseipeîn) a algo un color determinado, de modo de estar denominándolo correctamente (orthós prosagoreúein)? (182d1-5) ${ }^{22}$.

Lo primero que parece ponerse en cuestión es la capacidad de llamar o denominar (proseipeîn, prosagoréuein) correctamente una cosa por el hecho de que todo está sometido continuamente al flujo. Así, no es posible atribuir propiedad o naturaleza alguna a una cosa puesto que en el mismo lapso de la enunciación ella ya no contaría con la propiedad que se le atribuye $\mathrm{e}^{23}$. Considero que una premisa que opera implícitamente en este argumento, sin la cual resultaría incomprensible, es que todo cambio supone pasar de ser $\mathrm{x}$ a ser no $\mathrm{x}$ ( $\mathrm{y}$ por " $\mathrm{x}$ " debemos entender cualquier término del lenguaje). Esta polémica premisa es la que está en la base de

22 CORNFORd (1935 96-101) consideró que las cualidades a las que hace referencia aquí Platón son las formas. El argumento justamente apuntaría a mostrar que sin la estabilidad de las formas no podemos contar con una referencia para el lenguaje. OWEN (1965: 322 y ss.) en cierta medida retoma esta idea al considerar que la refutación del heraclitismo extremo constituye una demostración indirecta de la existencia de entidades de carácter no sensible, sin la cuales sería imposible un discurso acerca del ser. Mc Dowell (1973: 180-184), por su parte, aun admitiendo que la blancura no remite aquí a las formas, consideró que lo que en este contexto se establece es que el cambio afecta a la propiedad misma, a la blancura tomada en tanto universal. Bоsтоск (1988: 103-105) rechaza ambas posiciones y establece muy convincentemente que Sócrates refiere aquí al cambio de color de la cosa, es decir que la propiedad que cambia es la que se instancia u ocurre en la cosa. Considero que si uno atiende al contexto, la posición de Bоsтоск es la más sólida. En efecto, solamente ateniéndonos al breve pasaje citado, queda en claro que lo que está en continuo flujo es tò leukón (la cosa blanca, 182d1) y que en consecuencia al referir al flujo de la blancura (tês leukótetos, 182d2) no puede sino estar refiriendo al blanco de la cosa.

23 Esta concepción aparece desde la presentación misma de la tesis movilista en 152c-d. Allí se afirmaba: "nada es uno (hén) en sí y por sí mismo y no podrías darle una denominación adecuada ni decir que posee una determinada cualidad, pues si llamas a una cosa grande también parece pequeña, si la llamas pesada también parece liviana" (Teet. 152d2-5). Esta misma concepción acerca del modo en que deberíamos expresarnos atendiendo a la verdadera naturaleza de las cosas aparece en Teet. 157b3-8, texto en que se rechaza la posibilidad de admitir, entre otras, expresiones como "algo", "de alguien", "mío" porque indican fijeza y por tal razón quien las dijera sería fácilmente refutado.

ARgos 39 (2016) ISSN 0325-4194, pp. 9-32 
la imposiblidad de referir exitosamente a una cosa, pues al denominarla "blanca", por ejemplo, esta cosa ha pasado a ser no blanca ${ }^{24}$.

En 182d8-e2, el movilista admite que no sólo el objeto se mueve y cambia, sino también la percepción misma: el ver y el oír no permanecen ni un momento (póte, 182e1) siendo tales. Pareciera que la velocidad y la continuidad del movimiento desdibujaran el espesor temporal. O más bien parece desdibujarse la posibilidad de identificar instantes. Así, debemos decir que la visión cambia en no visión, de modo que ninguna cosa es visión más bien que no visión. El argumento implicaría que, puesto que, en el mínimo lapso de tiempo que podamos concebir, cualquier cosa que sea pasa de ser $\mathrm{x}$ a no $\mathrm{x}$, debemos admitir también que el acto perceptual de ver es también un no ver ${ }^{25}$. Así, puesto que la percepción [de algo] no es más percepción que no percepción, "cuando nos preguntábamos (erotómenoi) qué es el conocimiento respondíamos (apokrinámetha) que es algo que no es más conocimiento que no conocimiento" (182e11-12). Esta afirmación nos reintroduce, como en el pasaje en que Teodoro se refería a la imposibilidad de mantener un intercambio dialógico con el movilista, en un contexto dialéctico de preguntas y respuestas. Interesa destacar, además, que la pregunta a la que se hace referencia y que guía el diálogo, “¿qué es el conocimiento?”, implica la búsqueda de una definición. Para ser consecuente con su propia ontología, el movilista tendría que responder con algo que es y no es a la vez. Es por eso que, poco más adelante, Sócrates afirma:

Es evidente, según parece, que, si todas las cosas se mueven, cualquier respuesta (apókrisis) sobre cualquier cosa que se responda es igualmente correcta (homoíos orthè eînai). De modo que decir que las cosas son así

24 Al respecto cf. Bозтоск (1988: 105), quien afirma directamente que, puesto que este punto de partida es falso, el argumento es malo. De hecho, los errores en que han incurrido gran parte de los intérpretes surgirían del hecho de haber considerado que se trata de un buen argumento. Así, en el pasaje citado cuando Sócrates pregunta si es posible referir a un color, Teeteto claramente le tendría que haber respondido que sí. Que la cosa cambie de lugar, por supuesto, no impide denominarla correctamente. Y que vaya cambiando en sus cualidades tampoco impide un juicio verdadero acerca de la cosa porque, por un lado, uno puede afirmar que en el momento 11 x tenía tal propiedad y este ser un juicio verdadero, aun cuando en $\mathrm{t} 2$ dejara de tenerla. Por otro lado, que la cosa cambie por ejemplo en sus tonalidades de blanco no impide llamarla "blanca" durante un cierto lapso de tiempo.

25 No es el lugar para desarrollar un análisis pormenorizado de estos primeros pasos del argumento. Sólo me interesa mencionar que hay dos posibles razones para afirmar la imposibilidad de la permanencia de los actos perceptivos: una, porque "ver" se asume en tanto que ver $\mathrm{x} y$, en la medida que $\mathrm{x}$ pasa a ser no $\mathrm{x}$, se pasa de un ver $\mathrm{x}$ a un no ver $\mathrm{x}$. La segunda razón, más convincente quizás, es que todo acto, en tanto se encuentra entre las cosas que son, se ve sometido al flujo universal. 
y que no son así es igual o, si quieres, que llegan a ser (gígnesthai) para que no nos inmovilicemos con el discurso (183a4-8)

En este segundo momento del argumento se pasa de la rectitud o corrección del nombre aislado al de la respuesta. Lo que es imposible ahora es ofrecer una respuesta correcta, cualquiera sea la pregunta, justamente porque tambalea la posibilidad misma de una corrección o adecuación (orthótes) del discurso -que determina cómo son las cosas- a lo real. Cualquier respuesta será igualmente correcta e incorrecta, verdadera y falsa, en la medida en que la cosa cambia constantemente en todos sus respectos y, en consecuencia, es y no es eso. Aquí termina de sellarse la consabida vinculación entre la tesis del movilismo y la de la negación del principio de no contradicción. El lenguaje, tal como lo conocemos, no puede dar cuenta de esta realidad. Aun cuando nos sirvamos de un verbo como "llegar a ser" (gígnesthai) en vez de "ser" (échein) para no inmovilizar las cosas, dice Sócrates, nos estaríamos refiriendo a un "así" y un "no así" al atribuirle una naturaleza a la cosa. Es decir, estamos intentando establecer cómo son las cosas, pero desde los mismos parmámetros del movilista, no contamos con un lenguaje adecuado para ello. Es por eso que, por fin, Sócrates termina de sepultar al movilista al establecer que ni siquiera puede hablar en estos términos, porque si dijéramos que algo es así (hoútô) lo estaríamos excluyendo del flujo:

Pues los que dicen este discurso (toîs tòn lógon toûton légousin) deben establecer algún otro lenguaje (állen phonèn), ya que ahora no tienen expresiones (rhémata) para su propia hipótesis (pròs tèn autôn hupóthesin), tal como son las cosas ahora, a no ser que la expresión "ni siquiera así", dicha de un modo indefindo (ápeiron legómenon), se ajuste mejor a ellos. (183b2-5)

Se ha discutido en torno al hecho de que Sócrates esté admitiendo o no la posiblidad de un lenguaje alternativo, adecuado al movilismo. En efecto, podría pensarse que Sócrates está refiriéndose aquí a una especie de lenguaje peculiar, que habría intentado acuñar el movilista acorde a la naturaleza fluyente de todo lo que es. Según ha intentado mostrar Sedley, se podría pensar que desde los principios establecidos por el movilista, no todo lenguaje queda anulado, como pretenden las lecturas tradicionales, sino que el lenguaje se modifica para exhibir tal naturaleza fluyente y contradictoria. Así, el movilista rechazaría cualquier término que implique estabilidad y propiciaría un lenguaje que exhiba la contradicción y que se 
sirva de términos que impliquen un campo semántico amplio, ambiguo e indeterminado ${ }^{26}$.

En consecuencia, una vez más nos estaríamos enfrentando a un tipo de refutación que no apunta a inconsistencias internas en la posición del interlocutor, sino a una inconsistencia entre lo dicho y el hecho de formularlo en un ámbito dialéctico. Lo que colapsa, en palabras de Sedley, no es el lenguaje sino la dialéctica. En este sentido, el movilista no puede pretender estar dando cuenta de la naturaleza del conocimiento, sencillamente porque en su lenguaje no tienen espacio las nociones de verdad y falsedad. Él ya no podría presentar una auténtica hipótesis acerca del mundo, y en este sentido sus formulaciones ya no podrían tener el carácter de verdad que, en el fondo, pretenden. El movilista, sin embargo, aspiraba a presentar una respuesta a la pregunta acerca de qué es el conocimiento, de suerte que en la postulación misma de su tesis, razona Sócrates, se auto-refuta. Lo hace en la medida en que para formular su propia tesis, debe suponer un objeto estable que opere como referencia de aquello que se dice y esto es justamente lo que niega su tesis. Hemos visto que lo que se encontraba compometido primeramente en este argumento era la posibilidad del lenguaje de guardar una relación con su propio objeto. En un primer momento, en efecto, se atacaba la posibilidad de correspondencia o de adecuación entre los términos atómicos del lenguaje y lo real. Si lo real es puro flujo, los nombres ya no pueden establecer una relación de adecuación con ellos. Pero lo que se ponía en cuestión enseguida, sobre esta base de inadecuación de los nombres, era la posibilidad de ofrecer una respuesta correcta, de decir cómo son las cosas, que es justamente lo que está supuesto en el hecho de postular una hipótesis que pretende dar cuenta de la naturaleza de lo real, en este caso del conocimiento. Así, se encontaría comprometido aquí un segundo plano en el argumento, que implica la imposibilidad de dar cuenta a través del lenguaje de la estructura de lo real, como pretende hacer el movilista, en la medida que para tal cosa se re-

26 Al respecto los pasajes 152d2-6 y 157a7-b7 nos muestran que el heraclíteo propicia tal lenguaje. SEDLEY (2003: 96-98) defiende férrea y consistentemente esta posición y sostiene que son los heraclíteos mismos los que se auto-niegan el lenguaje. Lejos de colapsar, el lenguaje del movilista supondría sus propias reglas. El autor sospecha que esto se pudo haber dado efectivamente y que la figura de Crátilo a la que hacen referencia Platón y Aristóteles así lo atestiguaría. Habría existido, pues, una línea movilista que intentó acuñar un nuevo tipo de lenguaje que reflejara el movimiento y la consecuente contradicción a la que están sometidas las cosas. Esta corriente, por otro lado, según establece SEDLEY, encontraría un antecedente en ciertos fragmentos de Heráclito como B 32 y B 67 en que se exhibe un tipo de discurso que superpone contrarios y esquiva verbos como "ser". Contra esta posición cf. MARCOS (2014: 113 n. 32), quien afirma que "la vigencia de una tesis semejante para Platón está reñida con todo uso del lenguaje".

ARGos 39 (2016) ISSN 0325-4194, pp. 9-32 
quiere una cierta adecuación entre lo dicho y lo que es. De modo que si el adversario movilista quiere presentar una hipótesis, cualquiera sea, deberá reconocer una cierta estabilidad de lo real para que esto pueda oficiar de referente del discurso. Así, una vez más, encontramos comprometidos, como en el argumento anterior, los prerrequisitos dialécticos implicados en la postulación de la propia tesis.

III. La crítica final a la identificación entre aísthesis y epistéme (184b3-187a3)

Vamos por fin al controvertido argumento que esgrime Sócrates para refutar definitivamente la identificación planteada originariamente por Teeteto entre conocimiento (epistémen) y percepción (aisthesisis). Interesa exhibir una hasta aquí inadvertida relación posible en cuanto a la estrategia asumida con los otros dos argumentos mencionados.

A partir del argumento esgrimido contra el heraclitismo radical se ha rechazado que el conocimiento sea percepción sobre la base de la concepción heraclítea de la realidad, pero es necesario investigar ahora la percepción misma, para ver si sobre la base de una ontología diferente, la tesis de Teeteto es admisible.

El primer paso de la argumentación consiste en establecer que el hombre no ve con los ojos ni oye con los oídos (para ello se emplean dativos que pueden tener valor de agente o de instrumento), sino con el alma a través de ellos (utiliza aquí diá más genitivo). Esta distinción terminológica apunta a marcar la diferencia entre el agente que realiza la acción y aquello a través de lo cual se lleva a cabo. Así, percibimos los colores a través de los ojos y no con los ojos. Cada facultad (dúnamis), establece Sócrates a continuación, se dirige exclusivamente a un determinado tipo de objetos, y esto lo distingue de las otras facultades: lo que aprehende la vista no puede ser aprehendido por el oído y viceversa ${ }^{27}$.

Una vez establecido el rol instrumental de los sentidos, Sócrates afirma, a través de la célebre imagen del caballo de madera, la existencia de una naturaleza única que es el alma (psuché) "o como se la quiera llamar", con la que, a través de los sentidos como instrumentos (órgana), percibimos los objetos sensibles. Ahora bien, aun cuando cada órgano tiene un objeto que le es propio -el color del ojo, el sonido del oído-, es posible pensar (diánoûmai) algo de ambos al mismo tiempo, por ejemplo que ambos son (amphotéro estón). Y si esto es así, el ser no puede ser objeto de los órga-

27 Como señala Bostock (1988: 116), esta afirmación no se condice con la obvia posibilidad de aprehender cosas a través de varios sentidos. Esto sucedería, por ejemplo, con respecto a la forma o al movimiento. Al respecto cf. Aristóteles De anima III 1 425a13-b10. 
nos de los sentidos. Así, lo que es común (koinón) al objeto de más de un sentido no puede ser aprehendido a través de un sentido $u$ otro, pues no existe ningún órgano especial para percibirlo. De modo que el alma misma debe ser la que discierne los comunes que, como el ser, la semejanza, la diferencia, la identidad, la unidad, la belleza, la fealdad, la bondad o la maldad se aplican a los objetos de diversos sentidos (185a-186a) ${ }^{28}$. El argumento gira, entonces, en torno a las propiedades que les atribuimos a las cosas y, correlativamente, a las facultades (dunámeis) que nos las revelan (déloî, 185c5) ${ }^{29}$.

Ya establecida la diferenciación inicial entre objetos propios de los sentidos y objetos comunes a más de uno, Sócrates avanza en la caracterización de cada uno de ellos a partir de una investigación acerca del tipo de aprehensión que suponen. Hay entonces, según hemos visto, un tipo de propiedades a las que el alma accede a través de los sentidos. La capacidad de percibir impresiones de las propiedades que llegan al alma a través del cuerpo (dià toû sómatos pathémata), señala Sócrates, "se le presenta por naturaleza al hombre y a las bestias desde el momento mismo del nacimiento"(185b11-c1), de suerte que todo ser dotado de sentidos puede aprehender este tipo de objeto. Las meras impresiones (pathémata) suponen así afecciones, algo que le acontece al cuerpo: en estos casos el alma es meramente receptiva.

El segundo tipo de objeto, el de los comunes, no puede, en cambio, ser aprehendido con el auxilio de los sentidos, lo cual no significa que la apertura a los objetos acerca de los cuales se juzga no sea, en una primera instancia, a partir de aquellos ${ }^{30}$. En efecto, nuestro contacto originario con la cosa se dio a través de los órganos de los sentidos ${ }^{31}$. A la mera recep-

28 En torno al carácter de estos koiná se han generado interminables polémicas. Las posiciones oscilan entre aquellos que asumen que son las formas (es el caso de Cornford (1935: 102-109) o Guthrie (1978: 115)) u objetos abstractos y aquellos que, como Ryle (1939: 317), Crombie (1963: 14), Bondeson (1969: 114 y ss.) o Cooper (1970) entienden que tales comunes son considerados en este argumento meros predicados que se aplican a los objetos de más de un sentido, sin expedirse acerca de la naturaleza metafísica de los objetos a los que refieren.

29 Bostock (1988: 118) subraya el hecho de que en esta primera parte del argumento Platón no se refiere a juicios acerca de los objetos sensibles, sino a los objetos mismos, y es por eso que puede afirmar que cada sentido tiene su propio objeto, aún cuando en el juicio reconozcamos que una misma cosa puede ser percibida a través de varios sentidos.

30 Cabe señalar que el término "sképsasthai" se utiliza tanto con relación a lo aprehendido a través de los sentidos (el gusto, 185b7-c3) como con relación a lo común (186a10).

31 La perspectiva tradicional ha entendido que lo que establece Platón al distinguir entre aquello que se aprehende a través de los sentidos y aquello que el alma examina por sí misma implicaría una diferencia entre la percepción y el juicio. Considero que esta perspectiva genera muchos problemas a la luz del texto y que realmente no se exhibe en 
ción de las impresiones que atraviesan el cuerpo hacia el alma se le opone entonces otro tipo de proceso en que el alma es activa: las reflexiones (analogísmata) que ella lleva a cabo por sí misma. Así, frente a la percepción que supone la afección y la inmediatez del presente, en la reflexión (analogísma) interviene por necesidad el esfuerzo (mógis) y espesor temporal (en chróno). El alma examina las cosas percibidas una después de otra y las compara entre sí. Las reflexiones que menciona Sócrates en este contexto son en relación con el ser (ousía) y la utilidad (ophéleian) y, según afirma, quienes llegan a tenerlas sólo lo consiguen gracias a muchos esfuerzos y luego de un largo período de educación (186c2-5).

Por fin, Sócrates considera que está en posesión de todos los elementos que le permitirán refutar, finalmente, la tesis inicial de Teeteto según la cual la percepción se identifica con el conocimiento y ofrece un remate del argumento (186c7-186e). Hemos visto que conocemos algo, afirma, cuando reflexionamos (analogizomai) acerca del ser (ousía) y la utilidad (ophéleian) de algo. Puesto que la epistéme supone alcanzar la verdad y alcanzar la verdad de algo requiere alcanzar su ousía (186c7-10), y, según se ha establecido, tal aprehensión de la ousía no puede darse a partir de las meras impresiones sino por la reflexión, debemos concluir que la percepción (entendida como aquello que el alma realiza a través de los sentidos) no puede identificarse con la epistéme.

Vemos así que una vez más se acude a la noción de verdad para enfrentar al adversario. Sin embargo, esta no es entendida aquí como la relación de adecuación de un mero enunciado a la realidad, sino como el resultado de una reflexión en torno a la ousía, de modo que parece referirse más bien a la tarea que le compete al filósofo ${ }^{32}$. Mucho se ha discutido en torno tanto a la noción de común (koinón) como a la de ousía. Por supuesto, no intentaré zanjar esta discusión aquí, sólo me interesa reponer los elementos que permitan exhibir que una sutil consecuencia que se seguiría de este argumento es la imposibilidad, por parte de quien identifica percepción y conocimiento, de formular su hipótesis, en la medida que pretenda conferirle a ella misma un carácter verdadero. Según surge de la somera reconstrucción ofrecida del argumento, en él se desarrolla

él. Según entiendo, la diferencia relevante aquí no es entre percepción y juicio, sino entre propiedades asequibles a través de la percepción y propiedades asequibles a través del alma, sin mediación alguna de los sentidos (aun cuando, según hemos sugerido el material sobre la base del cual el alma juzga lo proporcionan los sentidos).

32 Si uno se atiene al excursus sobre la contraposición entre orador y filósofo de 172c-177c es evidente que la tarea de aprehender la ousía le compete al filósofo. En efecto, a este último "le interesa solamente alcanzar lo que es" (172e1). 
una doble caracterización de los koiná y, específicamente, del ser ${ }^{33}$. La primera mención que a ellos se refiere indica en dirección a toda propiedad que podemos atribuir al objeto de más de un sentido. Así, retomando al ejemplo brindado por Sócrates, el ser puede aplicarse tanto al color como al sonido. Este "ser" podría estar refiriendo tanto a la cópula, supuesta en cualquier predicación posible, ${ }^{34}$ como a la existencia ${ }^{35}$. Un segundo rasgo que se les atribuye a los koiná en esta primera parte del argumento es que no pueden ser aprehendidos con el concurso de los sentidos, como en el caso de los objetos propios, sino que el alma los alcanza "por sí misma" 36. Podríamos pensar entonces, como sugiere Sedley, que los koiná constituyen a priori, entendiendo aquí por tales aquellas entidades, predicados o conceptos, sean lo que fueren, que Sócrates considera que son alcanzados independientemente de la experiencia (aún cuando, según se evidencia en los ejemplos de Sócrates, los aplicamos a propiedades sensibles) ${ }^{37}$. Estas constituyen, por otro lado, aquello que Platón identificó tradicionalmente en tanto objetos de la investigación dialéctica ${ }^{38}$. Ahora bien, frente a las interpretaciones tradicionales, entiendo que los koiná no refieren directamente a "términos comunes" o "predicados comunes", como suponen

33 Como señala SEDLey (2004: 112), no sólo las nociones de "koinón" y "ousía" presentan tal ambigüedad. Esta se refleja correlativamente en las nociones de "alétheia" y "epistéme que aparecen hacia el final del argumento.

34 Así lo interpreta BuRnyeAt (1976b: 44-50). Respecto del hecho de que la cópula de ser está implícita en cualquier predicación posible cf. Bosтоск (1988: 131-132).

35 De hecho, según ha demostrado KAHN (1973), estos dos sentidos son inescindibles para el griego. Cf. también COOPER (1970: 140). Es cierto, sin embargo, que puesto que en 185c5-6 Sócrates señala que también el no ser se predica de todo, no puede ser la no-existencia lo que está en juego. Correlativamente habría que interpretar "ser", entonces, en un sentido amplio, que no excluya la existencia pero que no se reduzca a ella. Al respecto cf. Bostock (1988: 129) y SEDLEy (2004: 106 n. 162)

36 Como bien ha destacado CoOper (1970: 132), el hecho de que Platón intente combinar esta duplicidad de criterios a la hora de definir los comunes -poder atribuirse al objeto de más de un sentido sentidos y constituir objetos que el alma alcanza por sí misma- no parece contribuir a identificar una clase particular de objeto. Por otro lado, es particularmente llamativo el hecho de que Platón ejemplifique los comunes exclusivamente a través de su aplicación a propiedades sensibles de más de un sentido ("el color y el sonido son"), de modo que no resulta claro por qué deberíamos considerar que opera por sí misma al margen de los sentidos.

37 Creo que Sócrates deja ex professo indefinido el carácter metafísico de los koiná. En el texto no encontramos una alusión directa a las formas, aun cuando los pocos rasgos que les asigna coinciden, al menos parcialmente, con los que se atribuyen a las formas en los diálogos de madurez. Es claro que aquí lo que le interesa a Sócrates no es hacer foco en la naturaleza de estos comunes, sino solamente establecer una diferenciación entre ellos y los objetos de la percepción a partir de compromisos metafísicos que Platón pretende mínimos.

38 SEDLEY 2004: 106-107. 
algunos intérpretes ${ }^{39}$. Considero que Platón no traza tal identificación sin más: no está aludiendo directamente al plano lingüístico y esto se percibe con nitidez atendiendo al vocabulario empleado a lo largo del argumento. En efecto, en él Platón elude cuidadosamente cualquier referencia a las nociones vinculadas al juicio (dóxa) o al lenguaje (lógos). En este sentido, si uno se atiene a la profusión de términos vinculados con el lenguaje y con la predicación en el argumento inmediatamente anterior dirigido contra el movilista, salta a la vista que Platón no está pensando los koiná inmediatamente en tales términos ${ }^{40}$. Sin embargo, aun cuando el lenguaje no sea puesto en un primer plano en este argumento, es claro que Platón considera que la aprehensión de tales propiedades (o como se las quiera llamar) es retraducida por el hombre en términos lingüísticos ${ }^{41}$. Así, una primera consecuencia que se seguiría de su argumento es que la misma posibilidad de la predicación y la enunciación se ve comprometida a partir de la tesis del interlocutor, que reduce todo conocimiento a percepción. De modo que en su mismo empleo del lenguaje, el interlocutor estaría ex-

39 CoOPER, por ejemplo, considera que desde el comienzo del argumento lo que está en juego es el lenguaje y la capacidad de la predicación (1970: 128). Por su parte, SEDLEY (2004: 106) también se refiere a "predicados comunes" y lo justifica afirmando que en esta parte del diálogo estos comunes son considerados puramente en su "capacidad predicativa".

40 En este sentido, la interpretación propuesta se distancia tanto de la de aquellos que entienden que la distinción entre percepción y conocimiento supone aquella entre una instancia judicativa (la de los koiná) y una que no lo es (la de la percepción) (CoRnFord, BuRNYEAT, BOSTOCK), como de la de aquellos que, como CoOPER, consideran que lo que está en juego en el argumento son dos tipos de predicado: aquel que refiere al mero nivel de la percepción y que supone la mediación de los sentidos y aquel que supone la aprehensión de los koiná. Frente a ambas posiciones, encuentro que en el pasaje no se mencionan los términos "dóxa" ni "lógos" en el sentido de juicio. Llamativamente, Platón se cuida mucho de utilizar estos términos y cualquier otro referido al proceso de decir, llamar, predicar, enunciar, etc.. Al referirse a las operaciones realizadas en relación con los comunes utiliza términos que aluden al pensamiento y a sus procesos (skopeísthai y krínein, $186 \mathrm{a} 10$ y b78; en otras ocasiones para referir a lo que hace el alma con relación a los comunes utiliza lambánein, 185b8 o eporégesthai, 186e4). Es cierto, no obstante, que inmediatamente después de establecer las conclusiones del argumento Sócrates afirma que aquello a lo que se aboca el alma cuando se ocupa por sí misma de las cosas que son se denomina "opinar" (doxázein). Sin embargo, esta consideración abre una nueva argumentación que culminará también en una refutación, de modo que uno podría considerar que el error está en considerar justamente que aquello que hace el alma por sí misma es meramente juzgar. La captación de la ousía supuesta en todo conocimiento no podría identificarse entonces con el juzgar como han pretendido la mayoría de las lecturas ofrecidas.

41 Así se sigue de la línea 185c en que Sócrates le pregunta a Teeteto qué facultad exhibe lo que es común a todas las cosas "y a estas en particular a las que llamas "es" y "no es" ( hôi tò "éstin" eponomázeis kai tò "ouk ésti"). De modo que Platón sugiere aquí que en el lenguaje se refleja la aprehensión de estos comunes. 
hibiendo la imposibilidad de su propia tesis. Es importante subrayar que esta no es una consecuencia que el mismo Sócrates extraiga aquí de su análisis. Este recurso, sin embargo, sí será explotado en el Sofista a la hora de enfrentarse a diversos adversarios como el monista, o quien niega la posibilidad de decir una cosa de otra: en estos casos sí se les sustraerá el lenguaje, razón por la cual podemos pensar que Platón mismo irá explorando lentamente este recurso a la posibilidad del lenguaje como estrategia frente a adversarios radicales.

Ahora bien, en los últimos pasajes del argumento, los koiná "se robustecen" y son presentados en tanto objetos de los analogísmata. Implican entonces un conocimiento que no es inmediato ni se da en el caso de todos los hombres, de suerte que no puede tratarse aquí de la mera capacidad de predicar o de atribuir existencia, capacidad de la que estaría dotado todo hablante competente ${ }^{42}$. En efecto, si lo que se pone en juego en la aprehensión de estos comunes es la mera capacidad de juzgar, puesto que esta es alcanzada por todos los hombres y no supone esfuerzos a lo largo del tiempo como los descriptos por Sócrates, no se comprende la razón por la cual es considerada el resultado de un largo proceso de educación. Esta segunda caracterización que ofrece de los comunes tiene que ver ya más específicamente con las condiciones dialécticas. Hemos dicho que en la segunda parte de este argumento, Sócrates refiere a la ousía y a la utilidad como objetos de reflexión: lo que allí está en juego, como mencionábamos más arriba, no puede ser la mera capacidad de predicar ser del objeto de más de un sentido, sino el hecho de aprehender el carácter último de la cosa y volcar tal conocimiento en el ámbito de la práctica. En este sentido, como señala SEDLEY, tal búsqueda parece estar estrechamente emparentada con la emprendida por el Sócrates platónico desde los primeros diálogos. En efecto, esta indagación en torno a la ousía, al "qué es" de la cosa en cuestión, parece haber guiado la dialéctica socrático-platónica. En este sentido, si por verdad acá entendemos aprehensión del ser de la cosa, objeto último de la dialéctica, aquel que identifica conocimiento y percepción se sustrae a la aprehensión de toda ousía y, en consecuencia, de toda verdad. De modo que difícilmente podría pretender presentar su propia tesis como una verdad acerca del conocimiento. Así, quien se confine en el nivel de la percepción no podrá ofrecer definición ni del conocimiento ni de cosa alguna, pues cualquier definición supone la aprehensión de una ousía a la que el alma accede trabajosamente por sí misma, sin el concurso de los sentidos. Tal consecuencia, sin embargo, tampoco es extraída con nitidez por Sócrates al culminar la refutación. Esto

$\overline{42}$ Con respecto al robustecimiento de la noción de "ser" que se constataría a lo largo del argumento, cf. Sedley (2004: 109-110). 
puede deberse a lo desdibujada que se encuentra dialécticamente la figura del defensor de tal tesis. Es Teeteto el que propone tal definición sin convencimiento alguno y podemos sospechar que a esta altura difícilmente se animaría a defenderla. De modo que la debilidad del adversario no obliga a poner en juego recursos dialécticos tan sofisticados y radicales como en los dos casos anteriores que hemos analizado, el del relativista protogárico y el movilista radical.

Recapitulando, una primera y sutil arista del argumento, entonces, sería que el lenguaje mismo exhibe el recurso a una dimensión que trasciende todo aquello que los sentidos nos proporcionan. Un mínimo juicio, aun aquel que articule datos brindados por la sensación, p.e. "el pizarrón es verde", se vale de elementos que escapan a la sensación, vale decir de elementos a priori. De modo que en la misma estructura del lenguaje, en la enunciación de cualquier tesis, estarían operando estos comunes. Una segunda arista del argumento, inadvertida quizás por el mismo Platón, concierne a la pretensión dialéctica de la definición ofrecida. Esta se ofrece como una verdad y, sin embargo, no puede ser alcanzada por los mismos sentidos, que, sin embargo, se postulan como única vía posible de acceso al conocimiento. Así, si bien el argumento, a diferencia de los dos anteriores, no apunta directamente a exhibir la auto-refutación en que incurren los oponentes, la formulación misma de esta tesis también invalida su contenido, en primer lugar porque se vale del juicio que por necesidad recurre al ser (noción común) y, en segundo lugar, porque pretende erigirse en verdad, y la aprehensión de la verdad supone la de la ousía, lo cual también implica dejar atrás la percepción.

\section{Conclusiones}

A partir de los tres argumentos analizados, podemos identificar el patrón común que los atraviesa. En efecto, en todos ellos encontramos dos momentos diferenciados que nos conducen a pensar que el recurso a las condiciones regulativas del discurso constituyó para Platón una importantísima herramientas para delimitar el espacio dialéctico. El recurso a este principio aspira a dejar por fuera de la discusión a un adversario radicalizado que no se puede sujetar a las condiciones básicas implicadas en cualquier afirmación que pretenda medirse en la discusión.

Un primer rasgo en común a destacar es que en los tres casos, el punto de partida de la refutación es un factum que no puede ser negado por el interlocutor en la medida que quiera postular su propia tesis y presentarse como un interlocutor válido en la arena dialéctica. Es decir, de lo que se parte es del acto del adversario de proferir una tesis y presentarla en tanto que tal. 
El segundo punto a destacar respecto a las tres refutaciones es que en todas ellas se verifica el reenvío a la pretensión de verdad envuelta en la postulación de la propia tesis por parte del adversario. Es decir, la refutación de esta tesis reenvía no en la dirección de sus consecuencias, sino en la de sus prerrequisitos y se destaca la pretensión de verdad como el primero de ellos. Y en cada uno de los argumentos se verifica que tales condiciones implicarían la negación de la tesis defendida por el adversario. En el primer caso lo que se exhibe es que la verdad a la que aspira la tesis de Protágoras es una verdad simpliciter, de modo que si bien la tesis de la homo-mensura supondría la imposibilidad de tal tipo de verdad, ella es implícitamente afirmada en el acto mismo de su enunciación en un contexto dialéctico. En el segundo caso, se muestra que si bien la tesis del movilismo extremo niega la posibilidad de adscribir una naturaleza determinada a cualquier cosa que sea, la misma tesis se pretende verdadera en tanto aspira a exhibir la naturaleza de lo real. Por último, en el caso de la tesis de Teeteto que identifica conocimiento y percepción, la pretensión de verdad implicada en la búsqueda de la definición emprendida le sería vedada, pues su existencia supone la admisión de propiedades que jamás podrán ser alcanzadas por la percepción.

Vemos entonces que Platón se sirve en esta primera parte del diálogo de una estrategia de enorme valor filosófico, en la medida en que, según la interpretación ofrecida, en vez de deducir a partir de la tesis del adversario afirmaciones que exhiben alguna inconsistencia respecto de un marco de creencias por aquel admitido, reenvía en la dirección opuesta, al acudir a las condiciones mismas de la emisión de la propia tesis por parte del adversario ${ }^{43}$. La condición en la que aquí se apoya es la pretensión de verdad, que opera no como condición específica de la tesis formulada sino de su misma emisión. En otras palabras, la pretensión de verdad refiere al hecho de que toda tesis lanzada a la arena dialéctica es presentada al menos desde una cierta aspiración a dar cuenta de cómo son las cosas en realidad, de modo que compromete una referencia al mundo. Así, Platón radicaliza el élenchos en la medida en que no aspira a demostrar la falsedad de tales tesis acudiendo a aquello que se sigue de ellas sino a sus mismas condiciones. De este modo, lejos de exhibir inconsecuencias, pretende forzar al oponente a renunciar de antemano a su propia tesis a riesgo de exhibirla como una tesis falsa en la medida en que la formule en un contexto dialéctico ${ }^{44}$. En definitiva, Sócrates demuestra, a través de es-

43 Entiendo que se podría pensar que tal estrategia prefigura la de los argumentos trascendentales, que harán su aparición en el Sofista.

44 En este sentido entiendo que aquí en Teeteto, pero sobre todo en una serie de argumentos que se ofrecerán en Sofista, se ofrece una variante respecto de las refutaciones clásicas 
ta particular vía, que la sola postulación de una tesis supone la admisión de ciertas presunciones que operarían como marco mínimo para dotar de sentido a cualquier práctica discursiva con alguna pretensión de competir dialécticamente.

\section{Bibliografía}

\section{Ediciones y traducciones}

CHAPPELL, T. (2005) Reading Plato's Theaetetus, Indianapolis, Hackett, 2005

Duke, E. A., Hicken, W. F. And Nicoll, W. S. M. et al. (1995) Platonis Opera. vol. I, Oxford

Mc Dowell, J. (1973) Plato's Theaetetus, translated with notes, Oxford

Narcy, M. (1994) Platon, Théétète. Traduction inédite, introduction et notes, Paris

\section{Estudios y comentarios}

Bostock, D. (1988) Plato's Theaetetus, Oxford.

BuRNYEAT, M. F. (1976) "Protagoras and self-refutation in Plato's Theaetetus", The Philosophical Review, 85.

(1976b) "Plato on the Grammar of Perceiving", Classical Quarterly, N. S. 26, 29-51.

Castagnol, L. (2010), Ancient Self-Refutations: The Logic and History of the Self-Refutation Argument from Democritus to Agustine, Cambridge.

CoOpER, J. M.(1970) "Plato on Sense-Perception and Knowledge (Theaetetus 184-186)", Phronesis, Vol. 15 №2, pp. 123-146.

Cornford, F. M. (1935) Plato's Theory of Knowledge, Londres(Hay trad. castellana, Buenos Aires, 1968).

Crombie, I. M. (1963) An Examination of Plato's Doctrine: Plato on Knowledge and Reality, London.

Fine, G. (1994) "Protagorean Relativisms" en Cleary, John \& Wians, William (eds.) Proceedings of the Boston Area Colloquium in Ancient Philosophy, Lanham-N. York, pp. 211-243.

que podíamos encontrar en los primeros diálogos platónicos. Contra cf. Wilmet (1990:97), quien considera, en cambio, que en estos diálogos se hace más explícito el procedimiento de los primeros diálogos. 
IRWIN, T. (1977) "Plato's Heracliteanism", Philosophical Quarterly 27, pp. $1-13$.

Kahn, C. H. (1973) The verb "be" in Ancient Greece, Dordrecht.

LeE, M-K. (2005) Epistemology after Protagoras, Responses to Relativism in Plato, Aristotle and Democrit, Oxford.

MARcos, G. (1996) "La crítica de Platón al relativismo protagórico", Revista de Filosofía y Teoría Política 31/32, pp. 429-438.

- (2006) "En defensa de Platón. Notas al argumentos de autorefutación de Protágoras (Teeteto 171a-c)" en Conocimiento, realidad y relativismo, M. C. Di Gregori y M. A. Di Berardino (comps.), México. - (2014) "Platón, Heráclito y el movilista radical: críticas y apropiaciones", en G. Marcos y M. E. Díaz, El filósofo y sus adversarios en los escritos de Platón y Aristóteles, Buenos Aires.

NiкuLin, D. (2010) Dialectic and Dialogue, California.

Owen G. E. L. (1953) “The Place of theTimaeus in Plato's Dialogues”, Class. Quart. N. S. III p. 86.

Robinson, R. (1941) Plato's earlier dialectic, Ithaca, New York.

Sedley, D. (2004) The Midwife of Platonism: Text and Subtext on Plato's Theaetetus, Oxford.

Spangenberg, P. (2009) "Las críticas de Platón y Aristóteles a la phantasía de Protágoras", en G. Marcos y M. E. Díaz (eds.), El surgimiento de la phantasía en la Grecia clásica. Parecer y aparecer en Protágoras, Platón y Aristóteles, Buenos Aires, pp. 99-122.

Vlastos, G. (1956) Introduction to Plato's Protagoras, Indianapolis and New York.

WATERLOW, S. (1977) "Protágoras and Inconsistency: Theaetetus 171a6-c7", Archiv für Geschichte der Philosophie Bd. 59, pp. 19-36.

WILMET, R. (1990) "Platonic Forms and de Possibility of Language", Revue de Philosophie Ancienne VIII, no1, 97-118. 